\title{
Dielectric Properties of Microwave Absorbing Sheets Produced with Silicone and Polyaniline
}

\author{
Luiza de Castro Folgueras*, Mauro Angelo Alves, Mirabel Cerqueira Rezende \\ Divisão de Materiais, Instituto de Aeronáutica e Espaço, \\ Departamento de Ciência e Tecnologia Aeroespacial - DCTA, \\ Praça Marechal do Ar Eduardo Gomes, 50, São José dos Campos, SP, Brazil
}

Received: November 16, 2009; Revised: March 29, 2010

\begin{abstract}
The aim of this study was to produce sheets of microwave absorbing materials using conductive polyaniline dispersed in a silicone rubber matrix and to characterize the electromagnetic properties (absorption, transmission and reflection of electromagnetic energy; and electric permittivity and magnetic permeability) of these sheets in the X-band $(8-12 \mathrm{GHz})$. Two sheets were produced: one $2.80 \mathrm{~mm}$ thick and the other $4.39 \mathrm{~mm}$ thick. The thinner sheet absorbed incident microwave energy more efficiently, attenuating up to $88 \%$ of the incident electromagnetic energy. Also, calculations were performed in order to determine the electromagnetic parameters that optimize the absorbent properties of these sheets. These calculations showed that these materials can be combined and altered to produce absorbing materials with a wide range of absorbing characteristics.
\end{abstract}

Keywords: microwave absorbing material, conducting polyaniline, flexible sheets

\section{Introduction}

In general terms, it is possible to describe the interaction of an electromagnetic wave with microwave absorbing materials (RAM's) as a phenomenon where the electromagnetic energy is transformed into thermal energy ${ }^{1,2}$. According to the principle of energy conservation, the electromagnetic wave impinging on a material can be reflected, attenuated or transmitted through the material. The response of the material to the wave depends on its intrinsic characteristics ${ }^{3,4}$; the absorption of energy by the material does not necessarily means that the material will heat up, sometimes the opposite can occur, i. e., the material can cool down.

To produce a RAM, it is necessary to select a matrix material (insulating polymer, porous substrate, etc.) that will act as a support for an energy absorbing center. The absorbing center may consist of, for example, a conducting polymer with good mechanical and chemical properties and whose electrical conductivity can be modulated $^{5}$. The behavior of conducting polymers illuminated by electromagnetic radiation in the X-band $(8-12 \mathrm{GHz})$ has been studied to understand how the conductivity of these polymers affect the absorption of electromagnetic energy and how these materials can be used in the production of RAM's ${ }^{6-9}$.

The electric permittivity $(\varepsilon)$ and magnetic permeability $(\mu)$ are parameters related to the dielectric and magnetic properties of a material, and directly associated to their absorbing characteristics ${ }^{10-14}$. The relative permittivity and permeability are represented by Equations 1 and 2, respectively; the values of these parameters are calculated from the experimental values of the transmission and reflection coefficients of the material.

$$
\begin{aligned}
& \varepsilon_{\mathrm{r}}=\varepsilon^{\prime}+i \varepsilon^{\prime} \\
& \mu_{\mathrm{r}}=\mu^{\prime}+i \mu^{\prime}
\end{aligned}
$$

In these equations, the primed and double-primed symbols denote real and imaginary components. When the material is lossy, the permittivity and permeability of are complex and some of the incident electromagnetic energy is dissipated ${ }^{15,16}$. In the case of a magnetic material, losses are produced by changes in the alignment and rotation of the magnetization spin ${ }^{3,10,17,18}$. When an electromagnetic wave illuminates a dielectric material, there is the formation of electric dipoles which align in the direction of the applied field, and the dipole alignment is directly related to the absorption of electromagnetic energy ${ }^{10,13}$. The real component of the permittivity is associated with the ability of the material to store energy, while the complex component is responsible for energy dissipation. The use of polyaniline as an energy absorbing center allows the production of dielectric RAM's with negligible magnetic permeability. Using a simple physical model, one can say that dielectric materials behave as an electric circuit consisting of capacitors in parallel with resistors ${ }^{13,19,20}$.

The knowledge of how to process materials and combine components, additives, and polymer matrices are decisive factors in the final properties of the RAM ${ }^{21}$. Therefore, the aim of this work was to measure the dielectric parameters of sheets produced by mixing polyaniline with silicone rubber. The properties of the sheets were analyzed in the X-band in order to determine the transmission, absorption and reflection coefficients of the material and also its electric permittivity and magnetic permeability. Analytical calculations were performed to determine the absorbing characteristics of the sheets for different thicknesses and when used in combination.

\section{Materials and Methods}

\subsection{Chemical procedures}

The energy absorbing center (conductive polyaniline) of the RAM was processed first. This polymer was produced chemically and in laboratory scale. The conductive form of polyaniline (green color - emeraldine salt- powder form) was prepared from aniline and 
by the action of the oxidizing agent ammonium peroxydisulfate in an acidic reaction medium (dodecylbenzenesulfonic acid - DBSA). Following, the doped polyaniline powder was added to a matrix composed of either one of two types of silicone rubber, L9000 and RTV630 (GE Silicones). The mixture was homogenized by mechanical agitation, and the processed materials were poured into $30 \times 30 \mathrm{~cm}$ molds and dried at $70{ }^{\circ} \mathrm{C}$ overnight. Two different sheets were obtained; the sheet produced with L9000 silicone rubber had a thickness of $2.80 \mathrm{~mm}$, and the one produced with RTV630 silicone rubber was $4.39 \mathrm{~mm}$ thick.

\subsection{Electromagnetic evaluation}

The electromagnetic properties of the sheets were analyzed using the waveguide technique (closed system) in the frequency range 8 to $12 \mathrm{GHz}$ (X-band). A rectangular wave guide was coupled to a vector network analyzer (model 8510C, Agilent ${ }^{\circledR}$ Technologies) connected to an S-parameter test Hewlett-Packard ${ }^{\circledR} 8510 \mathrm{~A}(45 \mathrm{MHz}-26 \mathrm{GHz})$ and a synthesized frequency generator Hewlett-Packard ${ }^{\circledR} 8340 \mathrm{~B}$ $(45 \mathrm{MHz}-26 \mathrm{GHz})$. The setup used for the measurements is shown in Figure 1.Small samples (about $2 \mathrm{~cm}^{2}$ ) were cut from the sheets and inserted into the waveguide. The complex electromagnetic parameters, (permittivity and permeability) were obtained from the measured values of the S-parameters ${ }^{22}$ (reflection and transmission coefficients, $S_{11} / S_{22}$ and $S_{12} / S_{21}$, respectively) using a commercial software specifically designed for this task (Agilent ${ }^{\circledR}$ Technologies). The attenuation of the incident radiation by the RAM's was obtained from the difference between the reflectivity of an aluminum plate (reference material, alloy 2024) and that of the same aluminum plate covered with the RAM.

\subsection{Analytical formulas for reflectivity of RAM's.}

In the case of single-layer RAM's, a layer of absorbing material is placed in contact with a metal plate (Figure 2). The absorbing characteristics of the RAM can be varied by changing the thickness of the absorbing materials and their electromagnetic properties (permeability and permittivity).

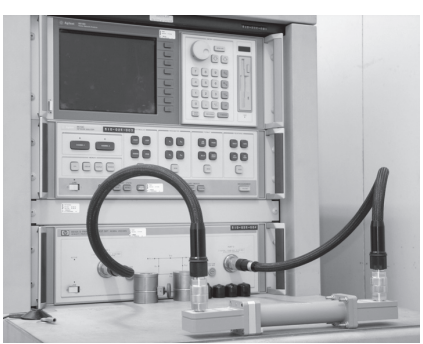

(a)

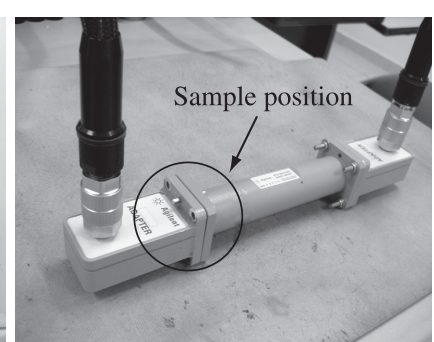

(b)
Figure 1. Electromagnetic measurement setup: (a) vector network analyzer, and (b) closed system rectangular waveguide and the position of the sample within the waveguide.

\begin{tabular}{|c|}
\hline$\varepsilon, \mu \quad$ RAM \\
\hline Metal plate \\
\hline
\end{tabular}

Figure 2. Single-layer RAM: a layer of a lossy absorbing material with complex permittivity and permeability $(\varepsilon$ and $\mu)$, and thickness $d$ was placed in contact with a metal plate.
The reflectivity of electromagnetic energy of a single-layer RAM as function of frequency can be calculated using the following equation ${ }^{10}$ :

$$
R(d B)=20 \log _{10}\left(\frac{i A \tan (k d)-1}{i A \tan (k d)+1}\right)
$$

where

$$
A=\sqrt{\frac{\mu}{\varepsilon}}, k=\frac{2 \pi f}{c} \sqrt{\mu \varepsilon}, i=\sqrt{-1}
$$

In the above equations, $\mu$ and $\varepsilon$ are, respectively, the complex permeability and permittivity of the absorbing material, $k$ is the wave number, $f$ is the frequency of the incident electromagnetic wave, $c$ is the speed of light in vacuum, and $d$ is the thickness of the absorbing layer. Both the permeability and permittivity may vary with frequency.

Two or more layers of absorbing material can be stacked (Figure 3) in order to improve the overall performance of the RAM.

The reflectivity of electromagnetic energy by a two-layer RAM can be calculated using the following equation ${ }^{10}$ :

$$
R(d b)=20 \log _{10}\left|\frac{\left[\frac{\left.\sqrt{\mu_{1} / \varepsilon_{1}} \tanh \left(\gamma_{1} d_{1}\right)+\sqrt{\mu_{2} / \varepsilon_{2}} \tanh \left(\gamma_{2} d_{2}\right)\right)}{\sqrt{\mu_{1} \varepsilon_{2} / \mu_{2} \varepsilon_{1}} \tanh \left(\gamma_{1} d_{1}\right) \tanh \left(\gamma_{2} d_{2}\right)}\right]-1}{\left[\frac{\left(\sqrt{\mu_{1} / \varepsilon_{1}} \tanh \left(\gamma_{1} d_{1}\right)+\sqrt{\mu_{2} / \varepsilon_{2}} \tanh \left(\gamma_{2} d_{2}\right)\right)}{\left(\sqrt{\mu_{1} \varepsilon_{2} / \mu_{2} \varepsilon_{1}} \tanh \left(\gamma_{1} d_{1}\right) \tanh \left(\gamma_{2} d_{2}\right)\right.}\right]+1}\right|
$$

where

$$
\gamma=i(2 \pi f / c) d \sqrt{\mu \varepsilon}
$$

\section{Results and Discussion}

In Figure 4 are shown the results derived from S-parameters measurements (reflected, transmitted and absorbed electromagnetic energy) as a function of frequency for the two single-layer RAM's produced in this study. Figures $4 \mathrm{a}$ and $\mathrm{b}$ show that both materials behave in a similar way; the absorbed, reflected and transmitted energies vary approximately linearly with frequency. Also, for both materials, the value of absorbed energy increased by about $10 \%$ with frequency in the frequency range of the measurements. The maximum energy absorption, which was measured at $8 \mathrm{GHz}$, was 18.5 and $16.2 \%$ for the materials with 2.80 and $4.39 \mathrm{~mm}$ respectively, showing that the thinner material had better absorbing properties. When the reflectivity of these materials were measured using an aluminum back plate (a situation similar to their use in the real world, i.e., RAM's are used to coat metallic objects) the $2.80 \mathrm{~mm}$ thick material absorbed $88 \%$ of the incident energy at $8 \mathrm{GHz}$ whereas the $4.39 \mathrm{~mm}$ thick material absorbed $71 \%$ of the incident energy this frequency. Lower values of absorption were obtained for higher frequencies. The behavior of both materials clearly indicates their potential for the production of RAM's, especially for civilian applications. The electromagnetic properties of the absorbing of these materials are shown in Figure 5.

\begin{tabular}{|lll|}
\hline$\varepsilon_{2}$ & $\mu_{2}$ & RAM \\
\hline$\varepsilon_{1}$ & $\mu_{1}$ & RAM \\
\hline & & Metal plate
\end{tabular}$d_{2}$

Figure 3. Two-layer RAM: absorbing materials with different electromagnetic properties are stacked together and placed in contact with a metal plate. 


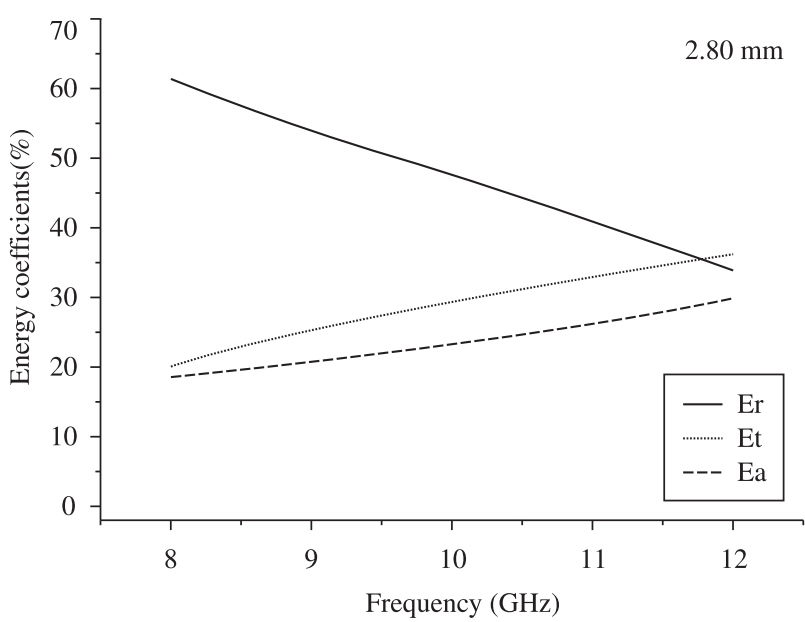

(a)

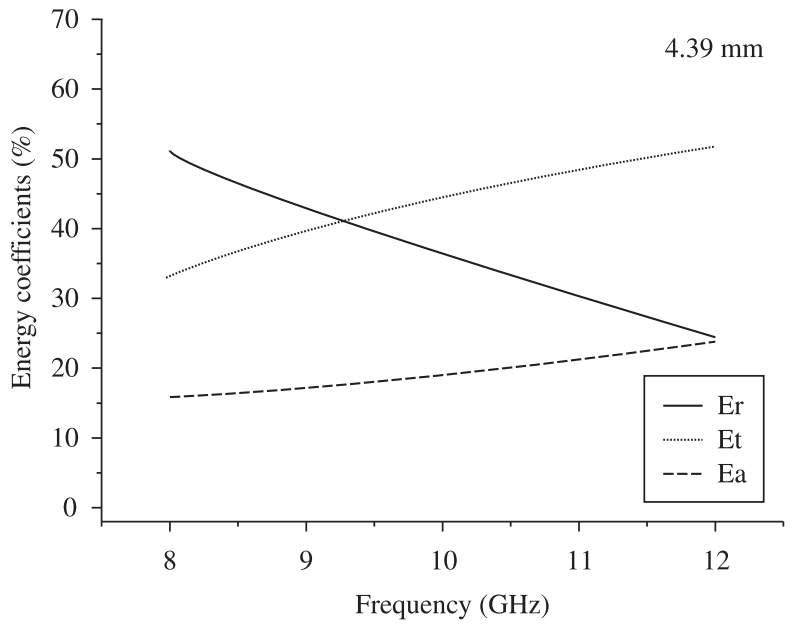

(b)

Figure 4. Curves of coefficients (in percentages) absorbed (Ea), transmitted (Et) and reflected (Er) energies: a) RAM thickness, $2.80 \mathrm{~mm}$, silicone matrix L9000; and b) RAM thickness $4.39 \mathrm{~mm}$, silicone matrix RTV630.

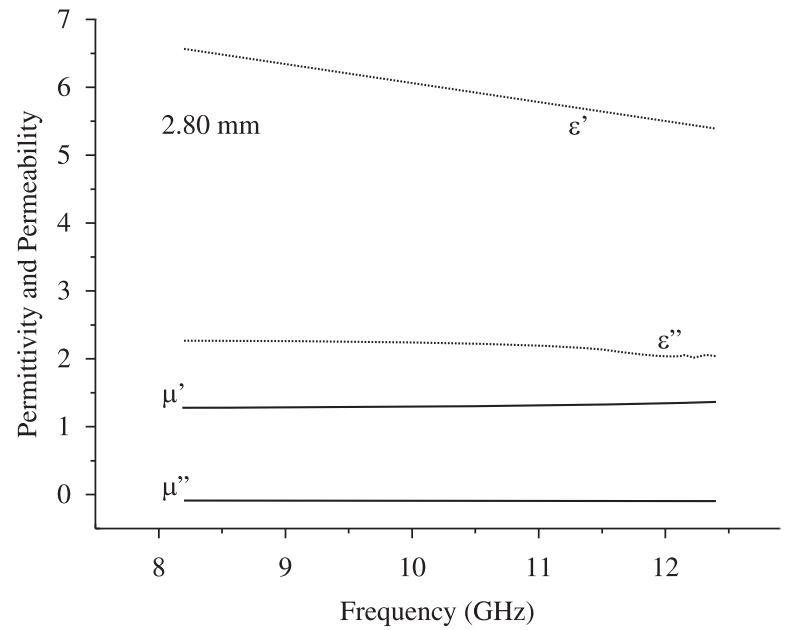

(a)

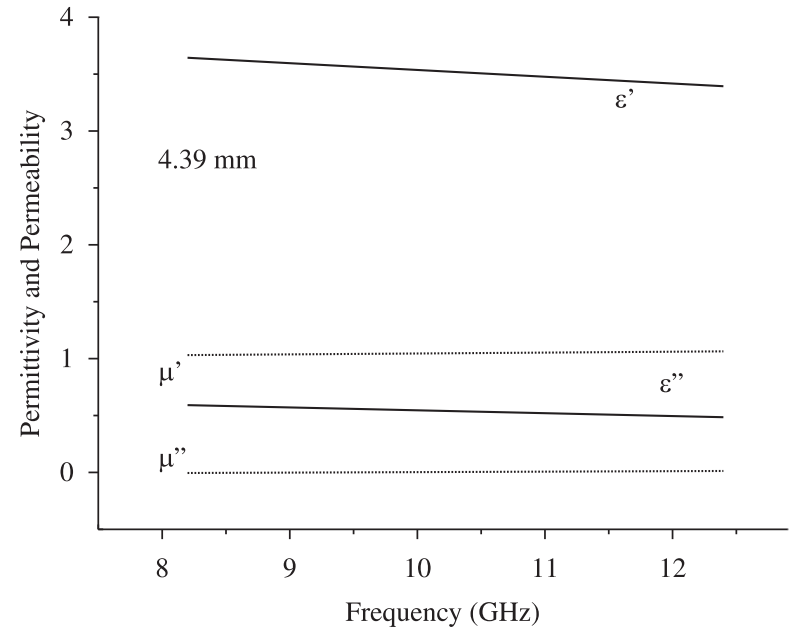

(b)

Figure 5. Complex relative permeability $\left(\mu \mathrm{r}=\mu^{\prime}+\mathrm{i} \mu\right.$ ") $)$ and relative permittivity $\left(\varepsilon r=\varepsilon\right.$ ' $\left.+\mathrm{i} \varepsilon^{\prime \prime}\right)$ of the absorbing materials: a) $2.80 \mathrm{~mm}$, silicone rubber L9000; and b) $4.39 \mathrm{~mm}$, silicone rubber RTV630.

These differences in behavior shown in Figure $5 \mathrm{a}$ and $\mathrm{b}$ are due to the different matrix compounds (silicon rubbers) used, since the absorbing center (conducting polyaniline) was the same for both materials. For the silicone rubber L9000 (Figure 5a), the real and imaginary values of the relative permittivity vary from 6.5 to 5.5 and from 2.1 to 1.85; for the silicone rubber RTV630 (Figure 5b) the real and imaginary relative permittivity vary little with frequency and their average values are about 3.5 and 5.0, respectively. As expected, since the materials were produced using dielectric components, the values of the real and imaginary magnetic relative permeability were approximately 1 and 0 , respectively, and did not show any major variation with respect to frequency.

By definition ${ }^{3,10}$, the real permittivity values are equal or larger than one. In an ideal material with zero dielectric loss, the real and imaginary permittivities are equal to 1 and 0 , respectively; in this situation the material does not store $\left(\varepsilon^{\prime}=1\right)$ nor dissipate $(\varepsilon "=0)$ energy. The larger the value of the imaginary component of permittivity the larger is the loss in the material. A material with low dielectric loss can store energy, but will not dissipate the stored energy. On the other hand, a material with high dielectric loss does not store energy efficiently and part of the energy of the incident wave is converted into heat within the material ${ }^{23}$.

Based on this information and on the results of Figures $5 \mathrm{a}$ and $\mathrm{b}$ one is led to the conclusion that the $2.80 \mathrm{~mm}$ thick material (silicone matrix L9000) is indeed has the best absorbing properties of the two RAM's produced in this study, as is evidenced by the results depicted in Figures $4 \mathrm{a}$ and $\mathrm{b}$.

Based on the experimental data (permittivity and permeability) and on Equation 3, it is possible to optimize the thickness of the RAM's with respect to the absorption of energy and its intended use. Figures $6 \mathrm{a}$ and $\mathrm{b}$ show how changes in the thickness affect the ability of the RAM's to absorb energy. The curves in these figures were obtained assuming that the RAM's were used to coat a flat metallic plate (Figure 2). Another interesting result shown in these figures 


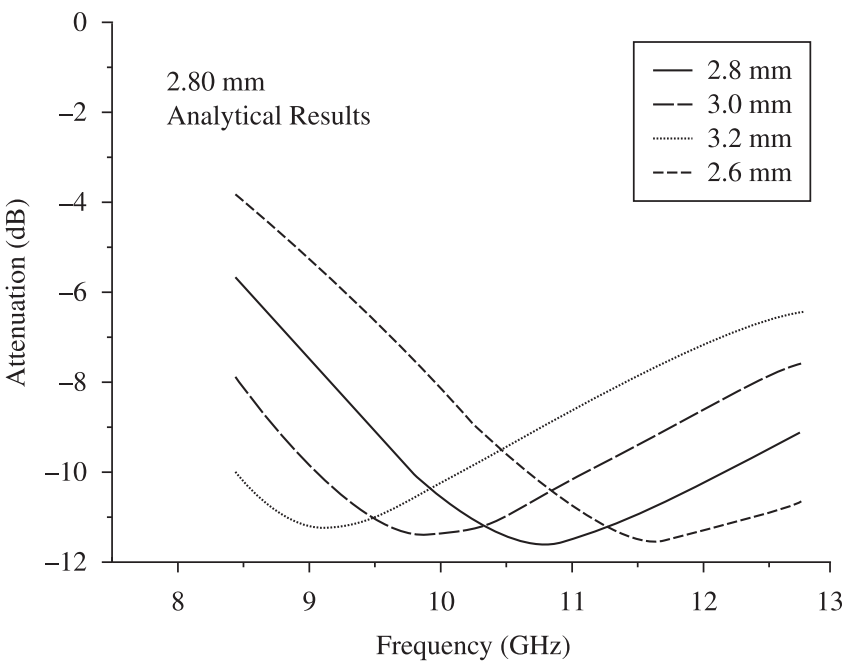

(a)

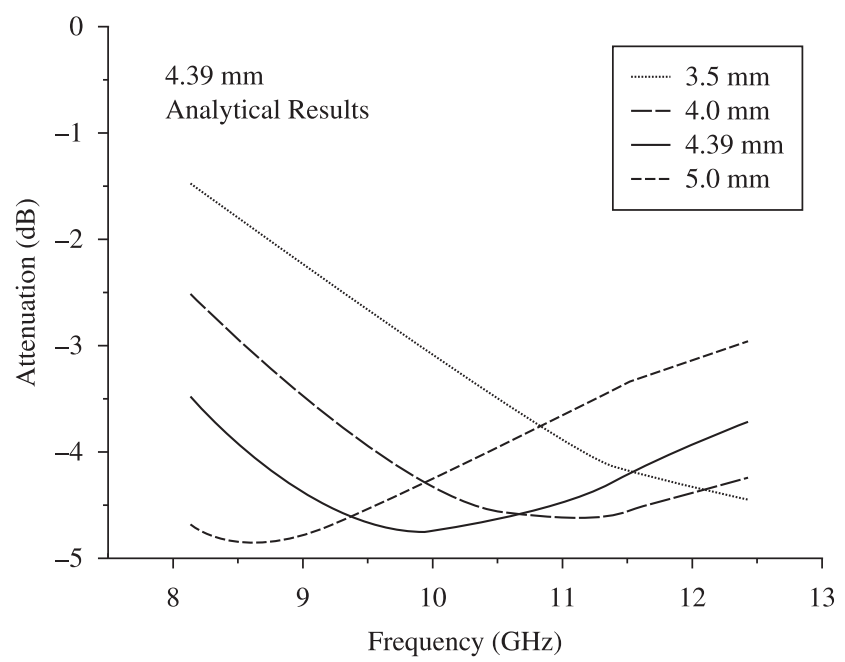

(b)

Figure 6. Energy absorption of single-layer RAMs as a function of frequency and different layer thicknesses: (a) RAM produced with silicone rubber L9000; and (b) RAM produced with silicone rubber RTV6300.

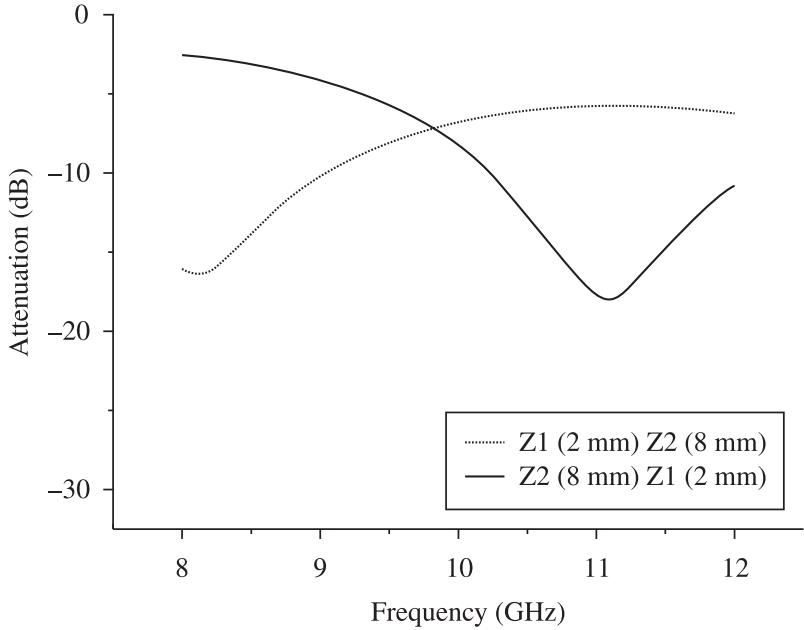

(a)

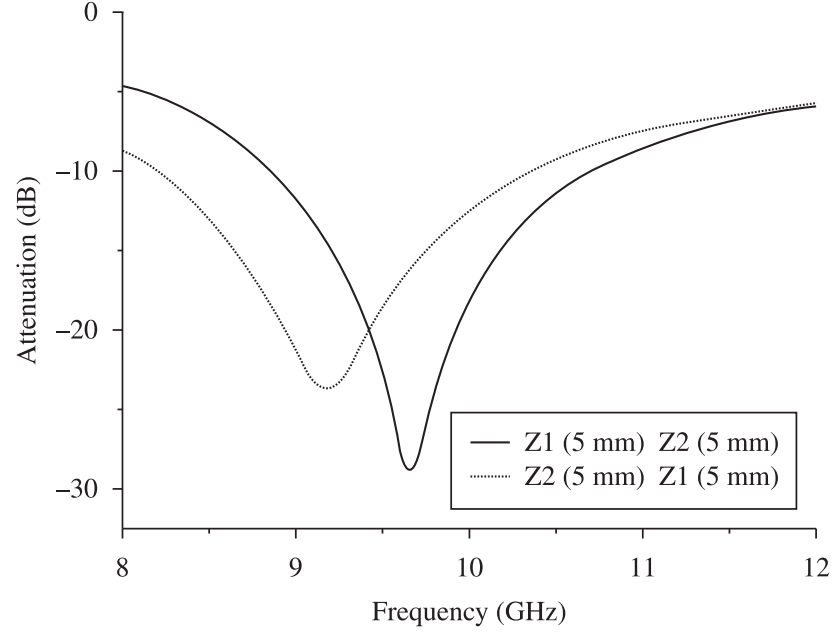

(b)

Figure 7. Simulations of two-layer RAM's. Attenuation of electromagnetic radiation as a function of frequency, thickness and stacking order of the absorbing materials. Z1, RAM produce with L9000 silicone rubber; Z2, RAM produce with RTC6300 silicone rubber: a) layers 2 and $8 \mathrm{~mm}$ thick, b) both layers are $5 \mathrm{~mm}$ thick.

is the displacement of resonance peak (maximum absorption) as a function of the thickness; note, however, that the absorption amplitude does not vary significantly. These calculations also show the RAM produced with the silicone rubber L9000 absorb electromagnetic energy more efficiently.

The single-layer materials developed in this study can be combined into multi-layer materials to produce RAM's with different absorbing properties. Using the experimental data collected on the single-layer materials, it is possible to simulate the behavior of two-layer RAM's using Equation 5. Figure 7 shows the results of simulations when these materials are combined in different configurations to produce a material with a total thickness of $10 \mathrm{~mm}$. In these curves, $\mathrm{Z} 1$ and $\mathrm{Z} 2$ refer to absorbing materials with the same electromagnetic properties of the materials prepared with silicone rubber type L9000 and RTC6300, respectively. The results in Figure 7a were obtained using 2 and $8 \mathrm{~mm}$ thick layers; in Figure 7b, both layers were $5 \mathrm{~mm}$ thick. It is interesting to observe that the order in which these materials are stacked plays an important role in determining the final properties of the RAM, affecting the amplitude and the position of the resonance peak.

\section{Conclusion}

The attenuation pattern of electromagnetic energy by the absorbing materials suggests that the electrical conductivity of these materials is related to the quantity of absorbing centers (conducting polyaniline) and type of polymer matrix (silicone rubber), which modify the impedance of absorbing materials. In absorbing materials, a large fraction of the incident energy must be attenuated, which is a consequence of the equilibrium between electric conductivity and electric losses. 
The materials produced with conductive polyaniline dispersed in a silicone matrix attenuated the incident radiation up to about $88 \%$, demonstrating that these materials can be used as absorbers of electromagnetic radiation. Also, the analytical calculations demonstrated the importance of optimization tools to produce absorbing materials with the required properties, since variations in the thickness or the combinations of materials with different properties can improve the absorbing qualities of the final material.

Another important characteristic of the materials produced using conducting polyaniline and silicone rubbers is their low density (no larger than $1 \mathrm{~g} \mathrm{g.cm}^{-3}$ ) compared with conventional absorbers based on ferrites (absorption $10 \mathrm{~dB}$ ), with densities ranging from 4 to 5 g.cm $\mathrm{cm}^{-3}$.

The RAM's produced in this study are light, flexible and have good absorbing properties; additionally they are relatively easy and inexpensive to produce. This set of characteristics demonstrates that these materials could be appropriate for a wide range of applications.

\section{Acknowledgment}

The authors acknowledge the financial support from CNPq (Procs. Numbers 559246/2008-0, 151803/2008-0 and 150048/2010-6), and the support received from the Departamento de Ciência e Tecnologia Aeroespacial.

\section{References}

1. Schleher DC. Electronic warfare in the information age. London: Artech House; 1999.

2. Folgueras LC and Rezende MC. Hybrid multilayer structures for use as microwave absorbing material. In: Proceedings of the IEEE 2007/ MTT-S International Microwave and Optoelectronics Conference; 2007; Salvador, Brazil. Salvador: IMOC; 2007. p. 483-487.

3. Kovetz A. Electromagnetic Theory. New York: Oxford; 2000.

4. Knott EF, Shaeffer JF and Tuley MT. Radar Cross Section. 2 ed. Norwood: Artech House; 1993.

5. Folgueras LC and Rezende MC. Multilayer radar absorbing material processing by using polymeric nonwoven and conducting polymer. Material Research. 2008; 11(3):245-249.

6. Hourquebie P and Olmedo L. Influence of structural parameters of conducting polymers on their microwave properties. Synthetic Metals. 1994; 65(1):19-26.

7. Faez R, Martin IM, Paoli MA and Rezende MC. Microwave properties of EPDM/PAni-DBSA blends. Synthetic Metals. 2001; 119(1):435-436.
8. Faez R, Rezende MC, Martin IM and Paoli MA. Polímeros condutores intrínsecos e seu potencial em blindagem de radiações eletromagnéticas. Polímeros: Ciência e Tecnologia. 2000; 10(3):130-137.

9. Bhadra S, Khastgir D, Singha NK and Lee JH. Progress in preparation, processing and applications of polyaniline. Progress in Polymer Science. 2009; 34:783-810.

10. Balanis CA. Advanced Engineering Electromagnetics. New York: John Wiley and Sons; 1989.

11. Lee SM. International Encyclopedia of Composites. New York: VCH Publishers; 1991.

12. Clark DE, Diane CF, Stephen JO and Richards S. Microwaves: Theory and Application in Materials Processing III. Westerville: The American Ceramic Society; 1995.

13. Hippel A. Dielectric Materials and Applications. London: Artech House; 1954.

14. Yusoffa AN, Abdullah MH, Ahmad SH, Jusoh SF, Mansor AA and Hamid SAA. Electromagnetic and absorption properties of some microwave absorbers. Journal of Applied Physics. 2002; 92(2):876-882.

15. Orfanidis SJ. Electromagnetic Waves and Antennas. [cited 2008 Oct.] Available from: <http://www.ece.rutgers.edu/ orfanidi/ewa/>.

16. Sucher M and Fox J. Handbook of the microwave measurements. 3 ed. New York: John Wiley and Sons; 1963.

17. Thostenson ET and Chou TW. Microwave processing: fundamentals and applications. Composites: Part A: Applied Science and Manufacturing. 1999; 30(9):1055-1071.

18. Jarem LM, Johnson JB and Scott W. Measuring the permittivity and permeability of sample at Ka Band using a partially filled waveguide. IEEE Transactions on Microwave and Techniques.1995; 43(12):2654-2667.

19. Venkatesh MS and Raghavan GS. An overview of microwave processing and dielectric properties of agri-food materials. biosystems engineering. 2004; 88(1):1-18.

20. Lakshmi K, Honey J, Rani J, George KE and Mathew KT. Comparison of microwave and electrical properties of selected conducting polymers. Microwave and Optical Technology Letters. 2008; 50(2):504-508.

21. Nicolaescu, I. Radar absorbing materials used for target camouflage. Journal of Optoelectronics and Advanced Material. 2006; 8(1):333-338.

22. American Society for Testing and Materials. Standard test method for measuring relative complex permittivity and relative magnetic permeability of solid materials at microwave frequencies using waveguide. West Conshohocken: ASTM; 2008.

23. Folgueras LC, Alves MA and Rezende MC. Development, Characterization and Optimization of Dielectric Absorbing Materials as Flexible Sheets for Use at X-band. In: Proceedings of the IEEE 2007-International Microwave and Optoelectronics Conference; 2007; Salvador, Brazil. Salvador: IMOC; 2007. p. 488-491. 